\title{
CASA Prediction System over Dallas-Fort Worth Urban Network: Blending of Nowcasting and High-Resolution Numerical Weather Prediction Model
}

\author{
CHANDRASEKAR RADHAKRISHNAN AND V. CHANDRASEKAR \\ Colorado State University, Fort Collins, Colorado
}

(Manuscript received 29 October 2018, in final form 29 November 2019)

\begin{abstract}
This study targeted improving Collaborative Adaptive Sensing of the Atmosphere's (CASA) 6-h lead time predictive ability by blending the radar-based nowcast with the NWP model over the Dallas-Fort Worth (DFW) urban radar network. This study also depicts the recent updates in CASA's real-time reflectivity nowcast system by assessing nine precipitation cases over the DFW urban region. CASA's nowcast framework displayed better primer outcomes than the WRF Model forecast for the lead time of $1 \mathrm{~h}$ and $30 \mathrm{~min}$. After that time, the predictive ability of the nowcast framework began decreasing compared to the WRF Model. To broaden CASA's predictive system lead time to $6 \mathrm{~h}$, the WRF Model forecasts were blended with Dynamic and Adaptive Radar Tracking of Storms (DARTS) nowcast. The HRRR model analysis was used as initial and boundary conditions in the WRF Model. The high-resolution dual-pol radar observations were assimilated into the WRF Model through the 3DVAR data assimilation technique. Three kinds of blending strategies were used and the results were compared: 1) hyperbolic tangent curve (HTW), 2) critical success index (CSIW), and 3) salient cross dissolve (Sal CD). The sensitivity studies were conducted to decide desirable parameters in the blending techniques. The outcomes proved that blending enhanced the prediction skills. Also, the overall performance of blending relies on the accuracy of the WRF forecast. Even though blending results are mixed, the HTW-based technique performed better than the other two techniques.
\end{abstract}

\section{Introduction}

With an expanding populace in urban territories, the precise forecast of extreme weather conditions is basic for sparing property and lives. The World Meteorological Organization (WMO) advises for nowcasting systems to be able to forecast severe weather conditions up to $6 \mathrm{~h}$ ahead (Wang 2017). The radar-based nowcasting methods, which use radar reflectivity or radar estimated quantitative precipitation estimation (QPE) fields, are more effective in shorter time spans. The radar-based nowcast strategies have an astoundingly higher predictive ability than numerical weather prediction (NWP) models over the initial hours. However, over longer times, the consistency of radar-based nowcasts are degraded because of the imperfections to determine the precipitation advancement (growth and dissipation) and uncertainty in assessing the storm motion fields (Turner et al. 2004).

With the aid of advanced computational facilities, the NWP model currently can also be utilized for

Corresponding author: Chandrasekar Radhakrishnan, chandrasekar. radhakrishnan@colostate.edu nowcasting. However, its significant limitations include the model spinup time and the elongated process to forecast, which makes outcomes less reliable. The NWP model physics parameterization schemes need a couple of hours to determine small-scale atmospheric features and dynamics. The model spinup time relies on the region and weather conditions. However, the model spinup time can be reduced through assimilation of high-resolution observations. The groundbased high-temporal- and high-spatial-resolution weather radar network observations are accessible in real time, which can be assimilated into the NWP models. Radar assimilation can diminish the uncertainty in the initial condition by adjusting the storm location and intensity. Even more sophisticated currently available NWP models with advanced data assimilation techniques, they are still not able to perform better than radarbased nowcasts for the first few hours. Blending the radar-based nowcast and NWP model predictions have the advantage of both and can extend the nowcast lead time with reliable accuracy.

This study focuses on extending the nowcast lead time by blending the radar-based nowcast and the NWP 
model prediction based on precipitation events in the Dallas-Fort Worth (DFW) area. This paper is organized as follows. Section 2 has an overview of radar-based nowcasting methodology and section 3 summarizes the Collaborative Adaptive Sensing of the Atmosphere (CASA), DFW's urban X-band dualpol weather radar network, and real-time nowcasting. Section 4 provides details of the WRF Model configuration and the 3DVAR assimilation technique. The experimental design and evaluation methodology are given in section 5 and the particulars of the blending methods are described in section 6. Section 7 discusses the findings, while the conclusions are given in section 8 .

\section{Overview of radar-based nowcasting methodology}

Existing radar-based nowcasting methods belong to one of the following five general attributes (or combination thereof): 1) object-based, 2) area-based, 3) statistical, 4) probability approaches, and 5) artificial intelligence (AI). The Royal Meteorological Institute (RMI) of Belgium (Reyniers 2008) listed and provided a detailed discussion of different types of nowcast methods. The object-based methods detect the areas of high reflectivity within radar fields and track shape and size, and translate the coherent features across time. This approach works best with strong, well-defined and structured storms. A few examples for object-based nowcasting include Storm Cell Identification and Tracking (SCIT; Johnson et al. 1998), the Thunderstorm Identification Tracking, Analysis, and Nowcasting (TITAN; Dixon and Wiener 1993), and TRACE3D (Handwerker 2002).

Area-based nowcasting methods estimate a motion vector over the entire radar coverage and extrapolate it into future time. These approaches not only track the storm's internal motion, but also signify the areal growth and decay of the storm. Area-based approaches need a particular shift in advection algorithm to evaluate the movement vector in order to deliver future fields from the most recent pastwatched information. The estimation of the distributed motion vector and advection process makes this strategy more computationally costly. Several examples of accessible area-based nowcast methods include Tracking of Radar Echoes by Correlation (TREC; Rinehart 1981; Rinehart and Garvey 1978; Tuttle and Foote 1990); Growth and Decay Storm Tracker (GDST; Wolfson et al. 1999); the McGill Algorithm for Precipitation Nowcasting by Lagrangian Extrapolation (MAPLE; Germann and Zawadzki 2002); the Integrated Nowcasting through Comprehensive Analysis (INCA) System from Zentralanstalt für Meteorologie und Geodynamik (ZAMG) (Haiden et al. 2011); and the Short-Range Warning of Intense Rainstorms in Localized Systems (SWIRLS) from the Hong Kong Observatory (Woo and Wong 2017).

In statistical nowcasting approaches, the atmospheric evolution is represented by statistical models that can consolidate the qualities of atmospheric dynamics. The statistical method most often utilizes the radial (Cornford 2004) or parameterized (Xu et al. 2005) elliptical basis functions to model the radar fields. The Bayesian-based Markov chain Monte Carlo (MCMC) model predicts the future radar fields, where mean posterior distribution is taken as nowcast and the standard deviation is considered as nowcast uncertainty. Another class of statistical nowcasting is called spectral prognosis (S-PROG; Seed 2003), where a notch filter is used to disintegrate the radar reflectivity into a cascade of arbitrary fields in the recurrence space. The nowcasts are generated by extrapolating each level in the cascade by a single motion vector that is projected by maximizing cross correlation over similar levels in successive radar fields.

The likelihood nowcast (Germann and Zawadzki 2004; Megenhardt et al. 2004; Schmeits et al. 2008; Dance et al. 2010) methods assess the probability of precipitation rates over a specific limit at every matrix focus over a radar convergence for a given lead time. The ensemble nowcast approach (Atencia and Zawadzki 2015; Foresti et al. 2016; Sokol et al. 2017) is currently the most popular probabilistic nowcast. The AI-based nowcast methods (Xingjian et al. 2015; Shi et al. 2017; Heye et al. 2017) have gained popularity in recent years. Machine learning (ML) approaches utilize the radar echoes and NWP model forecast data to train a network that can predict severe weather systems for a very short lead time. This is the least developed method at this point but has intriguing potential.

\section{Overview of the CASA's real-time nowcast system over DFW urban area}

\section{a. CASA DFW dense urban dual-pol weather radar network}

The DFW metroplex is a major inland metropolitan area and one of the fastest growing urban areas in the United States. It is home to the active DFW International Airport and several regional airports. The dense urbanization and populace requires an urban weather disaster detection and mitigation, and 


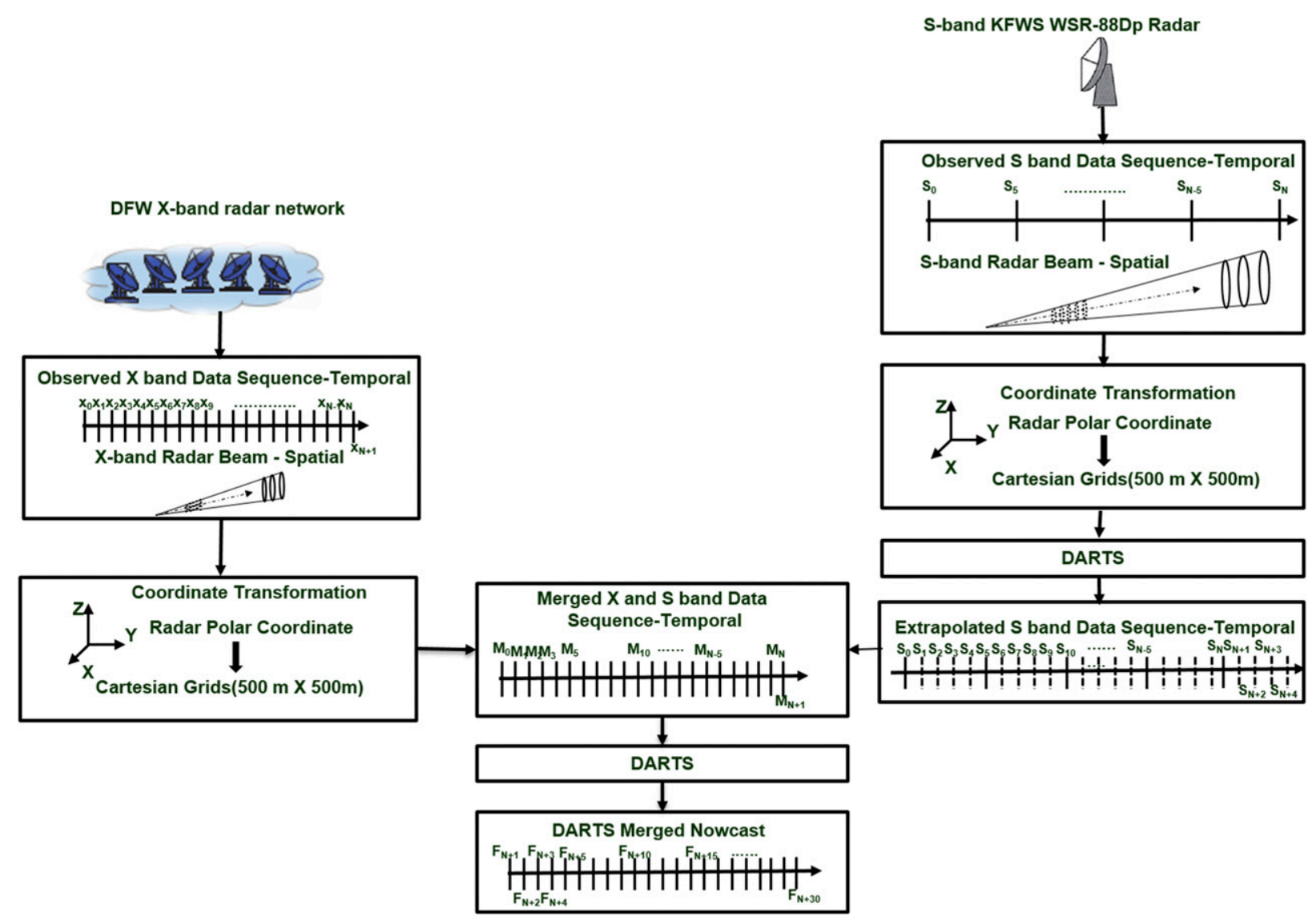

FIG. 1. CASA real-time operational reflectivity nowcast system data flowchart.

also provides an ideal place to demonstrate the dense ground-based weather radar network. CASA has installed the demonstration dense radar network over the DFW urban area with a network of eight X-band dual-pol weather radar locations. The geographic locations, technical specifications, and the detailed radar operations are given in Chandrasekar et al. (2018). CASA also created advanced algorithms to provide real-time products like QPE, wind fields and hail detection (Chandrasekar et al. 2018). Further, CASA also has the real-time high-resolution reflectivity and QPE nowcast over the DFW urban radar network. The Dynamic and Adaptive Radar Tracking of Storms (DARTS) nowcast tool was developed at CASA to meet the effective nowcasting requirement of CASA's Distributed Collaborative Adaptive Sensing (DCAS) system. DARTS is an area-based nowcasting tool and the advection is performed by an efficient sinc kernel representation of the reflectivity field. This procedure augments the performance of the DARTS system to predict storm motion, areal evolution and position using the high temporal and spatial resolution of CASA's radar data. The mathematical formulation for the DARTS model can be found from Ruzanski et al. (2011).

\section{b. Real-time nowcast system data flow and architecture}

The previous CASA nowcast system used radar observations either from CASA's DFW X-band network or NEXRAD's KFWS (Istok et al. 2009) S-band radar. The new nowcast system over the DFW urban area described in this paper has added the capability to merge both X-band and S-band radar reflectivity and delivers the merged reflectivity nowcast. The new system has been delivering the real-time nowcast since March 2018. The system has been well tested since implementation and proven to be robust. The system is intended to be very flexible and it can provide the nowcast even when one radar network data is unavailable. The nowcast system covers the $78400 \mathrm{~km}^{2}$ area over the DFW urban area with a grid resolution of $250 \mathrm{~m}$. Figure 1 shows the data flow of the CASA's new reflectivity nowcast system. The temporal resolution of the CASA X-band radar network merged reflectivity is every $1 \mathrm{~min}$, but NEXRAD KFWS radar reflectivity observations are 
available every $5 \mathrm{~min}$. To update the nowcast cycle for every $1 \mathrm{~min}$, it is necessary to get the NEXRAD KFWS radar observations into 1-min temporal resolution. To create the 1-min temporal resolution of NEXRAD KFWS radar observations, separate modules intended to execute the DARTS are used to extrapolate NEXRAD KFWS radar observations. This module runs the DARTS nowcast at 5-min intervals and gives the nowcast for the next $5 \mathrm{~min}$ with the most recent NEXRAD KFWS radar observations. The nowcast outputs are written every $1 \mathrm{~min}$ to fill the observations between two consecutive NEXRAD KFWS radar observations. After matching the temporal resolution of CASA's X-band and KFWS S-band radar observations, both network radar reflectivities are merged. The DARTS is executed with the merged reflectivity to provide a merged nowcast. The nowcast updates every $1 \mathrm{~min}$ and provides prediction for the next $30 \mathrm{~min}$.

\section{NWP model and data assimilation}

WRF, version 3.9.1 (Powers et al. 2017), was used in this study to simulate storms over the DFW urban area. The model domain had $301 \times 301$ horizontal grid points with a $1-\mathrm{km}$ spacing of the grid and 51 vertical levels between the surface and $50 \mathrm{hPa}$. The physics parameterization included: the WRF double-moment 6-class (WDM6) scheme for microphysics processes, the MYNN surface layer, the RUC land surface, the MYNN 2.5-level TKE planetary boundary layer scheme, the Rapid Radiative Transfer Model (RRTM) longwave radiation, and the RRTMG shortwave radiation scheme. The National Oceanic and Atmospheric Administration (NOAA) real-time 3-km-resolution, cloud-resolving, hourly updated convection-allowing High-Resolution Rapid Refresh (HRRR) model analysis (Benjamin et al. 2016; Blaylock et al. 2017) was used as the initial and boundary condition for the 1-km-resolution WRF Model. The WRF-3DVAR technique was used to assimilate both reflectivity and radial velocity observations from high-resolution CASA X-band and NEXRAD KFWS S-band radars into the WRF Model. Since the error in radar observations is not exactly known, a random error with a zero mean was generated from normal distribution and added to the radar observations. The standard deviation of $1 \mathrm{~m} \mathrm{~s}^{-1}$ and $3 \mathrm{~dB} Z$ was used to generate a random error for radial velocity and reflectivity data. The recent version of 3DVAR (3.9.1) offers the ability to assimilate hydrometers (Gao and Stensrud 2012) derived from the reflectivity data observed by radar. In this technique, the hydrometers are categorized according to
TABLE 1. Precipitation events over DFW urban area.

\begin{tabular}{ccccl}
\hline \hline No. & Date & $\begin{array}{c}\text { Start time } \\
\text { (UTC) }\end{array}$ & $\begin{array}{c}\text { End time } \\
\text { (UTC) }\end{array}$ & Event type \\
\hline 1 & 2 Apr 2017 & 0500 & 0800 & Line \\
2 & 10 Apr 2017 & 1900 & 2200 & Line \\
3 & 17 Apr 2017 & 1800 & 2100 & Supercell \\
4 & 21 May 2017 & 0900 & 1200 & Multicell \\
5 & 2-3 Jun 2017 & 2100 & 0000 & Supercell \\
6 & 3 Jun 2017 & 0600 & 0900 & Supercell \\
7 & 5 Jun 2017 & 1800 & 2100 & Supercell \\
8 & 9 Jun 2017 & 1000 & 1300 & Multicell \\
9 & 1 Jul 2017 & 1100 & 1400 & Supercell \\
\hline
\end{tabular}

the background temperature profile of the NWP model and the rain (QRAIN), snow (QSNOW), and graupel mixing ratios (QGRAUP), are used as control variables $(\mathrm{CV})$. To use the individual hydrometeor as the control variables during 3DVAR minimum, the cloud control variable (cloudcV) options were set as 3 . Thirty days of forecast ensemble members were put together to create a domain-dependent CV7 background error (BE) covariance matrix using the National Meteorological Center (NMC) method (Parrish and Derber 1992). In the WRF-3DVAR framework, the CV7 is one of the available BE options. As control variables, it uses $U$ wind, $V$ wind, temperature $(T)$, pseudo-relative humidity ( $\mathrm{RH}$ ), and surface pressure (Ps). In the CV7 BE, the control variables are in the eigenvector space and the vertical covariance is represented by an empirical orthogonal function (EOF).

\section{Experimental design and evaluation methodology}

Nine precipitation events were dissected in urban areas of the DFW metro and were then categorized into three types of events: 1) supercell, 2) line, and 3) multicell. The supercell weather event is a less common thunderstorm characterized by the presence of mesocyclones (a deep, persistently rotating updraft). It has an organized internal structure with strong vertical wind shear. A supercell can exist for several hours and has the potential for severe weather events. The line event (also referred to as the squall line) is a type of mesoscale convective system forming along or on a cold front. Damaging winds, large hail and tornadoes can be produced. The multicell is a cluster of cells that move as one unit and each cell represents different phases of the life cycle of the thunderstorm. The multicell storms can have a higher severity, and most flash floods occur in this type of storm.

A 3-h time window was selected within the life expectancy of each event; the date of the event, along with the start and end times are listed in Table 1. 


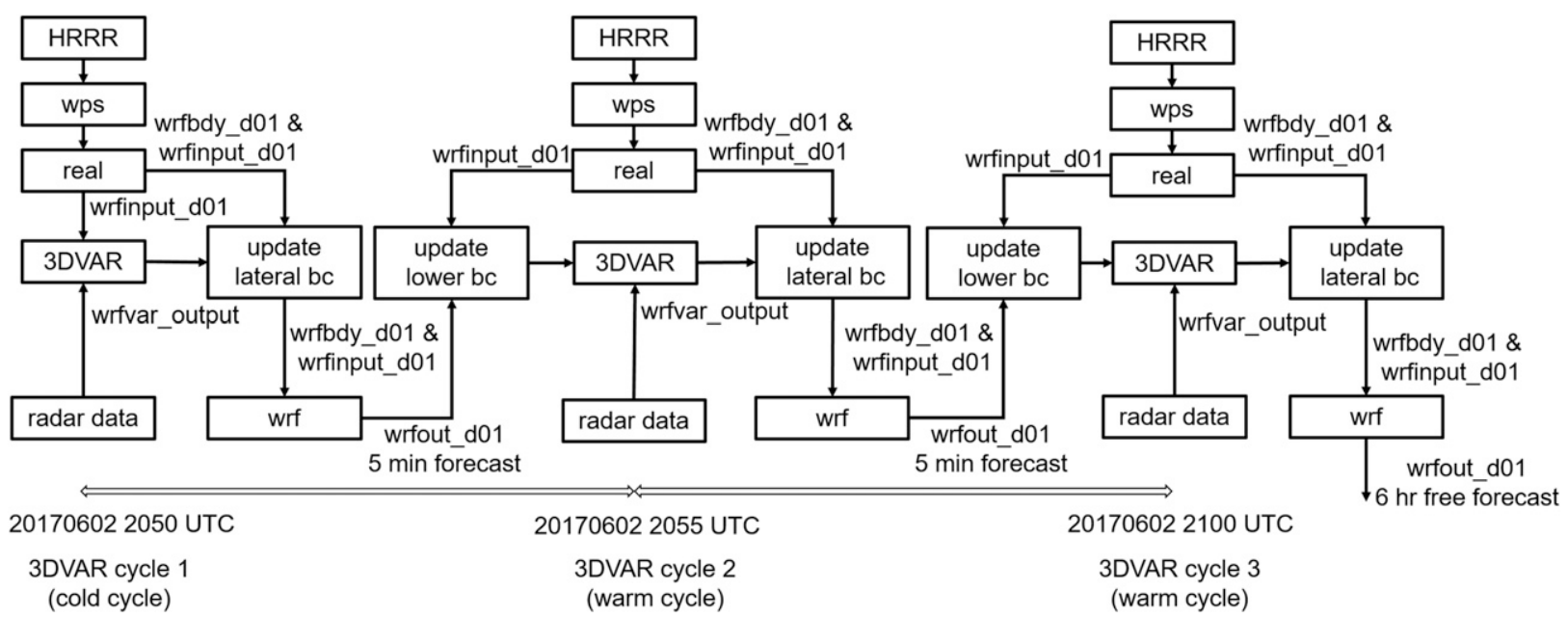

FIG. 2. 3DVAR assimilation cycles procedure used in 2 Jun 2017 precipitation event simulation. The event start time was 2100 UTC and assimilation cycles started $10 \mathrm{~min}$ before the start time and three cycles were used.

CASA's operational nowcast system offers the nowcast for $30 \mathrm{~min}$ in advance and refreshes every minute. For assessment purposes here, DARTS nowcast cycles were updated every 5 min within the chosen time window of each precipitation event; for a single event, a total of 36 nowcast cycles were generated. The first set of assessments were performed on DARTS with observed merged reflectivity. The skill scores are estimated for a single DARTS nowcast cycle and averaged for a particular event over all 36 cycles. The skill scores were averaged again over the specific type of precipitation.

The WRF Model was initialized from the HRRR model analysis and the selected storm events were simulated for a 6-h lead time. The WRF-3DVAR assimilation initializes another set of WRF simulations, which includes both NEXRAD KFWS S-band and CASA X-band radar reflectivity and radial velocity data. Both WRF Control (no assimilation) and WRF3DVAR simulations were initialized at the start time of the event listed in Table 1. This study used the assimilation system, with three assimilation cycles (one cold start and two warm starts) to estimate the best analysis state for the start time of the event. The first cold start cycle (the first guess for 3DVAR comes from the HRRR model analysis) starts $10 \mathrm{~min}$ prior to the start time of the event. A 5-min WRF forecast was made with best analysis estimated from the cold start cycle. Two warm start cycles (the first guess for 3DVAR comes from the previous 5-min WRF forecast, initialized with the best analysis estimated by cold start cycle) are made after the cold start cycle in 5-min intervals to estimate the best analysis state for the start time of an event. After this, a free WRF forecast is provided for the next $6 \mathrm{~h}$ with the best analysis estimated at the start time of the event.
Figure 2 shows the assimilation cycles procedure followed in this study. For all precipitation events, a 6-h DARTS nowcast was generated simultaneously when WRF was initiated. Following this, $6 \mathrm{~h}$ of DARTS nowcast was blended with the simulation of the WRF-3DVAR.

The second set of assessments was carried out between the predicted reflectivity fields of DARTS nowcast, HRRR, WRF control, and WRF-3DVAR model. All model predictions and observed merged reflectivity were regridded into HRRR model resolution $(3 \mathrm{~km})$ for this evaluation. The regrid function of the Earth System Modeling Framework (ESMF) from the NCAR Command Language (NCL) software (Brown et al. 2012) was used to regrid the DARTS nowcast, WRF simulations and the radar observation data into the HRRR grid. The bilinear interpolation method was used in the regrid function of ESMF. The third part of the evaluation took place among the DARTS nowcasting, the WRF-3DVAR predictions, and three blending techniques. The DARTS nowcast and the radar observations were regridded to WRF Model resolution $(1 \mathrm{~km})$ for the evaluation using the ESMF regrid function. To evaluate the performance of the forecast, the critical success index (CSI), probability of detection (POD), equitable threat score (ETS), and mean absolute error (MAE) skill scores were used. Equations (1)-(4) show the mathematical formulation of these skill scores:

$$
\begin{aligned}
\mathrm{CSI} & =\frac{n_{11}}{n_{11}+n_{10}+n_{01}}, \\
\mathrm{POD} & =\frac{n_{11}}{n_{11}+n_{01}},
\end{aligned}
$$




$$
\begin{aligned}
\mathrm{ETS} & =\frac{n_{11}-C_{1}}{n_{11}+n_{10}+n_{01}-C_{1},} \\
C_{1} & =\frac{\left(n_{11}+n_{10}\right)\left(n_{11}+n_{01}\right)}{T}, \\
\mathrm{MAE} & =\frac{1}{N_{i=1}^{N}\left|F_{i}-O_{i}\right|,}
\end{aligned}
$$

where $n_{11}$ is the intersection of the area over which the event was forecast and subsequently observed, $n_{10}$ is the area over which the event was forecast and subsequently not observed; $n_{01}$ is the area in which the event was observed but was not forecast to occur. Here, $N$ is the total number of forecast-observation pairs. The CSI and POD scores vary from 0 to 1 and the ETS score varies between $-1 / 3$ and 1 . For a perfect forecast the CSI, POD, and ETS scores will be 1, and the score 0 indicates no skill; $F_{i}, O_{i}$ is the $i$ th of $N$ pairs of forecasts and observations. The $20 \mathrm{dBZ}$ threshold value was used to estimate all skill scores.

\section{Blending radar-based nowcast and NWP model predictions}

This section presents various blending methods used to blend the radar nowcast and the NWP model forecast.

\section{a. HTW method}

The hyperbolic tangent curve weight (HTW) is the most commonly used method for blending NWP and radar nowcast (Wang et al. 2015; Wong et al. 2006; Wong et al. 2009). The inputs are weighted based on the function of time; with higher weights given to radar extrapolation for the first few hours and the weights are decreased exponentially with longer lead time. In the meantime, the NWP model weights are low for the first few hours of lead time, but are increased exponentially with increased lead time. The weights of the NWP model $\left(W_{\mathrm{NWP}}\right)$ estimated by Eq. (5) and the final blended reflectivity $\left(\right.$ Blend $\left._{\text {ref }}\right)$ can be calculated from Eq. (6):

$$
W_{\mathrm{NWP}}=\alpha+\frac{\beta-\alpha}{2} \times\left\{1+\mathrm{TANH}\left[\lambda_{1} \times\left(T-\lambda_{2}\right)\right]\right\},
$$

$$
\begin{aligned}
\text { Blend }_{\text {ref }}= & W_{\text {NWP }} \times\left(\mathrm{NWP}_{\text {ref }}\right)+\left(1-W_{\mathrm{NWP}}\right) \\
& \times\left(\operatorname{RadEx}_{\mathrm{ref}}\right) .
\end{aligned}
$$

In Eq. (5), the $\alpha, \beta, \lambda_{1}$, and $\lambda_{2}$ are tuning parameters and mainly depend on forecast lead time $(t)$ and type of weather events. The $\alpha$ and $\beta$ represent the NWP model
TABLE 2. Desirable parameters for hyperbolic tangent curve weight (HTW) method determined from sensitivity studies.

\begin{tabular}{lccrrrrrrrl}
\hline \hline Event type & $\alpha$ & $\beta$ & $\lambda_{1}^{\mathrm{S} 1}$ & $\lambda_{1}^{\mathrm{S} 2}$ & $\lambda_{1}^{\mathrm{S} 3}$ & $\lambda_{1}$ & $\lambda_{2}^{\mathrm{S} 1}$ & $\lambda_{2}^{\mathrm{S} 2}$ & $\lambda_{2}^{\mathrm{S} 3}$ & $\lambda_{2}$ \\
\hline Supercell & 0 & 1 & 5 & 6 & 7 & 5 & 1.3 & 1.4 & 1.5 & 1.5 \\
Line & 0 & 1 & 9 & 10 & 11 & 10 & 0.1 & 0.15 & 0.25 & 0.25 \\
Multicell & 0 & 1 & 13 & 14 & 15 & 15 & 0.1 & 0.166 & 0.2 & 0.166 \\
\hline
\end{tabular}

weights at forecasting lead time 0 and $6 \mathrm{~h}$. The $\lambda_{1}$ represents the slope in the middle of the blend and $\lambda_{2}$ represents the lead time when the $W_{\mathrm{NWP}}$ and radar nowcast weight $\left(W_{\text {RadEx }}\right)$ are equal $\left(W_{\mathrm{NWP}}=W_{\mathrm{RadEx}}=0.5\right)$. The optimum set of tuning parameters needs to be determined to get better blending. In Eq. (6), the $\mathrm{NWP}_{\text {ref }}$ and $\mathrm{RadEx}_{\text {ref }}$ represents the predicted reflectivity from the NWP model and radar nowcast. The initial assumption of these four parameters came from the DARTS model verification during the analysis of this study.

\section{b. CSIW method}

In the critical success index weight (CSIW) method, the radar nowcast weight is valued through the past performance of the particular nowcast model (Kober et al. 2012; Kilambi and Zawadzki 2005). The performance of a radar nowcast tool depends on geographical location factors. For example, the performance of the nowcast tool in a region of the continental United States (CONUS) will differ from a region in India region. Therefore, it is essential to estimate a nowcast tool's performance to a particular region. Then, the nowcast tool's performance can be converted into skillbased weights for that particular region and used when blending with a NWP model. In the CSIW method, the skill-based weights of radar extrapolation (RadEx) estimated from CSI score in Eq. (7). The CSI score of radar extrapolation is used in Eq. (7) to estimate the weights of radar extrapolation (RadEx). The value of power constant $(\tau)$ in Eq. (7) will be chosen in such a way that both the radar extrapolation and NWP model will get equal weight at a particular lead time. The desired value of $\tau$ will vary between different precipitation types. The final blended reflectivity based on the CSIW method can be calculated from Eq. (8):

$$
\begin{aligned}
W_{\text {RadEx }}= & \left(\operatorname{CSI}_{\text {RadEx }}\right)^{\tau}, \\
\text { Blend }_{\text {ref }}= & W_{\text {RadEx }} \times\left(\operatorname{RadEx}_{\text {ref }}\right)+\left(1-W_{\text {RadEx }}\right) \\
& \times\left(\mathrm{NWP}_{\text {ref }}\right) .
\end{aligned}
$$

\section{c. Sal CD weight method}

The weights estimated from the HTW and CSIW methods are a function of only time and are spatially 

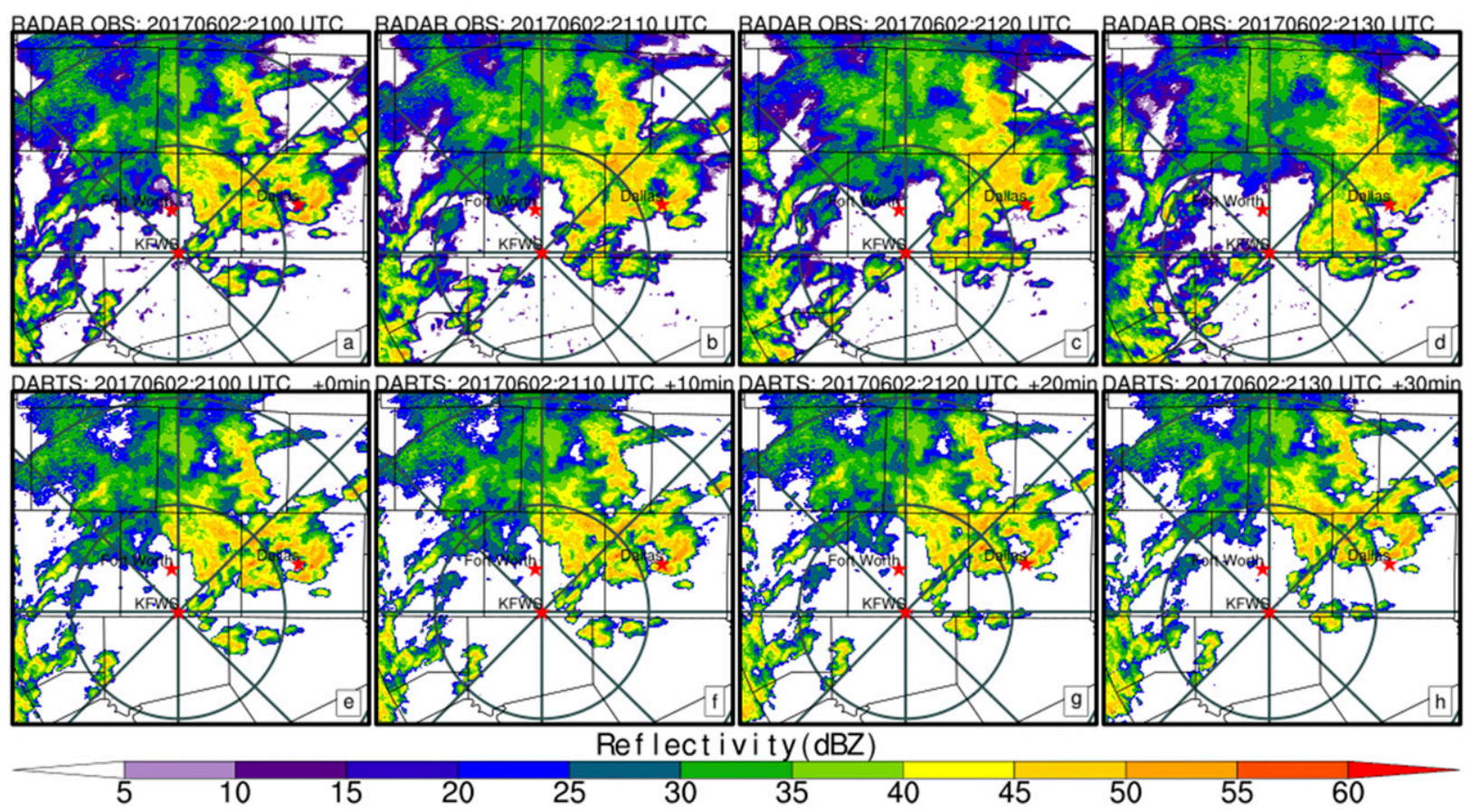

FIG. 3. (a)-(d) CASA and NEXRAD KFWS radar merged observed reflectivity and (e)-(h) DARTS nowcast reflectivity. (a),(e) Observed reflectivity at nowcast start time, and (b)-(d) and (f)-(h) the observed and nowcast reflectivity for the lead time of 10, 20, and $30 \mathrm{~min}$.

uniform (constant in every grid point of the nowcast domain). The blended reflectivity from these two methods underestimates the intensity of the storm. This is due to the fact the radar-based nowcast and the NWP model are weighed nearly equal, they have a spatial shift in the predicted reflectivity between the two models. This has been called the "half intensity" issue. This limitation can be overcome by using the salient cross dissolve (Sal CD) weighting method (Hwang et al. 2015). In the Sal CD method, the weights are a function of time and intensity:

$$
\begin{aligned}
& W_{s}\left(W_{\mathrm{NWP}}, I\right)=\frac{1}{2}\left[\frac{W_{\mathrm{NWP}} I}{W_{\mathrm{NWP}} I+\left(1-W_{\mathrm{NWP}}\right)(1-I)}+\frac{\sqrt{I^{2}+W_{\mathrm{NWP}}^{2}}}{\sqrt{I^{2}+W_{\mathrm{NWP}}^{2}}+\sqrt{(1-I)^{2}+\left(1-w_{\mathrm{NWP}}\right)^{2}}}\right], \\
& I(x, y)=\Phi\left[N_{\mathrm{NWP}}(x, y)-N_{\mathrm{RadEx}}(x, y)\right], \\
& \begin{aligned}
\text { Blend }_{\mathrm{ref}}= & W_{s}\left[W_{\mathrm{NWP}}, I(x, y)\right] \times \mathrm{NWP}_{\mathrm{ref}} \\
& +\left\{1-W_{s}\left[W_{\mathrm{NWP}}, I(x, y)\right]\right\} \times \operatorname{RadEx}_{\mathrm{ref}} .
\end{aligned}
\end{aligned}
$$



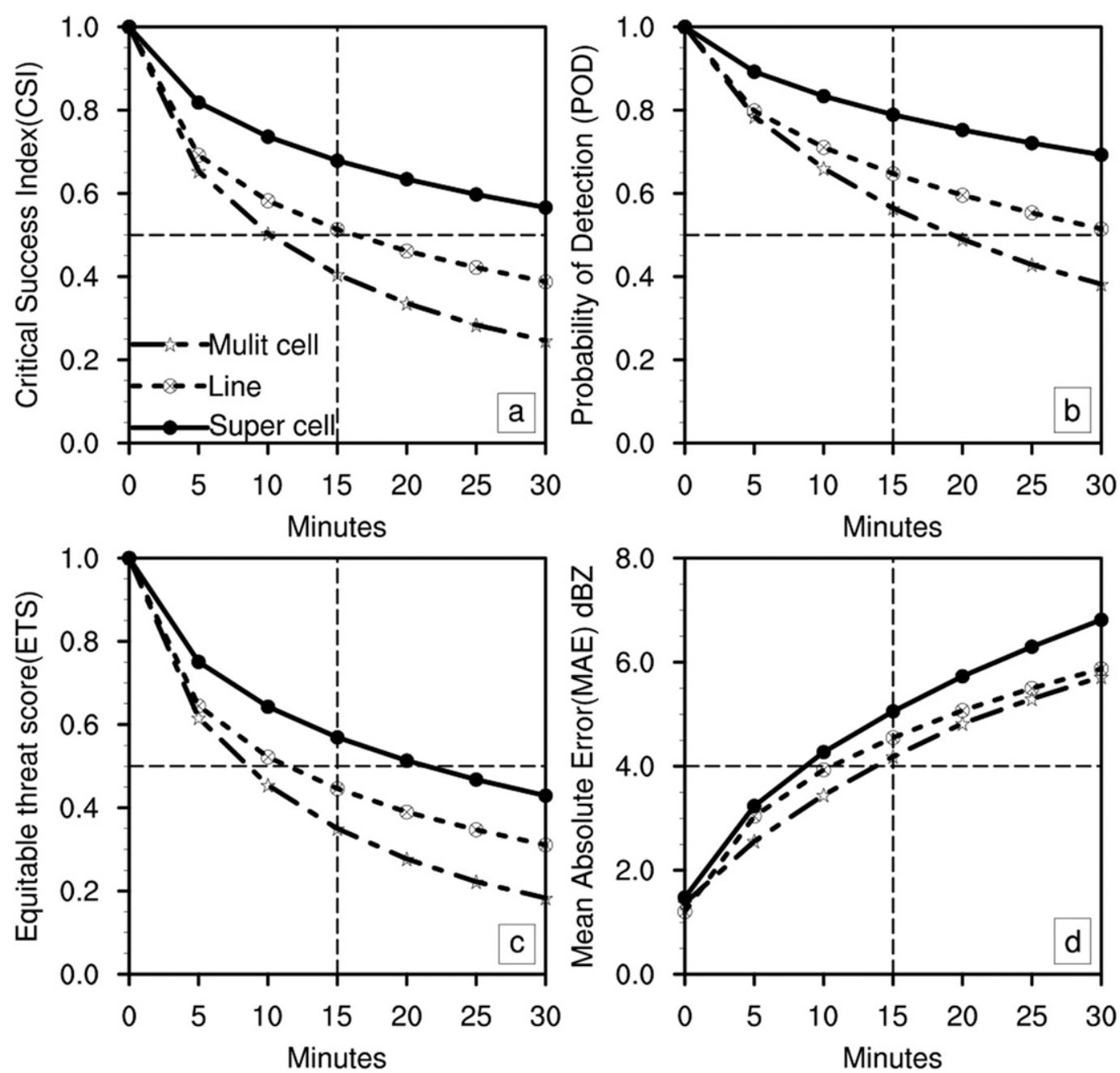

FIG. 4. Nowcast cycles and events averaged skill scores for three precipitation type 1) supercell events, 2) line events, and 3) multicell events.

\section{d. Sensitivity studies to determine the desirable parameters in blending}

In HTW and Sal CD blending techniques, the timedependent weights are estimated by Eq. (5). As discussed in section 6a, there are four parameters $\left(\alpha, \beta, \lambda_{1}\right.$, $\lambda_{2}$ ) in Eq. (5) and the desirable values of these parameters must be determined in order to get the accurate weights for the NWP model. The parameters are mainly dependent on forecast lead time $(t)$ and type of weather event. The $\alpha$ in Eq. (5) refers to the weight of the NWP model at lead time $(t)$ equal to 0 . Since DARTS has excellent performance at $t=0$ hours, the $\alpha$ is fixed to 0 for all the events during blending. The $\beta$ in the Eq. (5) refers to the weight of the NWP model at $t=6 \mathrm{~h}$. Since DARTS has low skill at $t=6 \mathrm{~h}$, the $\beta$ is fixed to be 1 for all types of weather events. After fixing the $\alpha$ and $\beta$, the desirable values of the remaining two parameters $\lambda_{1}$ and $\lambda_{2}$ are determined by sensitivity deigned for this study. Based on the performance of DARTS in the nine precipitation events considered in this study, three initial assumptions of the $\lambda_{1}$ and $\lambda_{2}$ were chosen for supercell, line, and multicell events. These initial assumptions for $\lambda_{1}\left(\lambda_{1}^{s 1}, \lambda_{1}^{s 2}, \lambda_{1}^{s 3}\right)$ and $\lambda_{2}\left(\lambda_{2}^{s 1}, \lambda_{2}^{s 2}, \lambda_{2}^{s 3}\right)$ are listed in Table 2 . Nine simulations were conducted for every precipitation event with three initial assumptions for $\lambda_{1}$ and $\lambda_{2}$. In total, 45 simulations were conducted for supercell events and 18 simulations for each were conducted for line and multicell events. The CSI scores were estimated for all simulations and the final values of $\lambda_{1}$ and $\lambda_{2}$ were taken from a simulation that has the higher CSI score on that particular type of weather event simulations. Since the number of precipitation events were less over DFW during chosen the time period, all events were used in the sensitivity study to estimate the desirable parameters. The desirable parameters were not tested on independent 

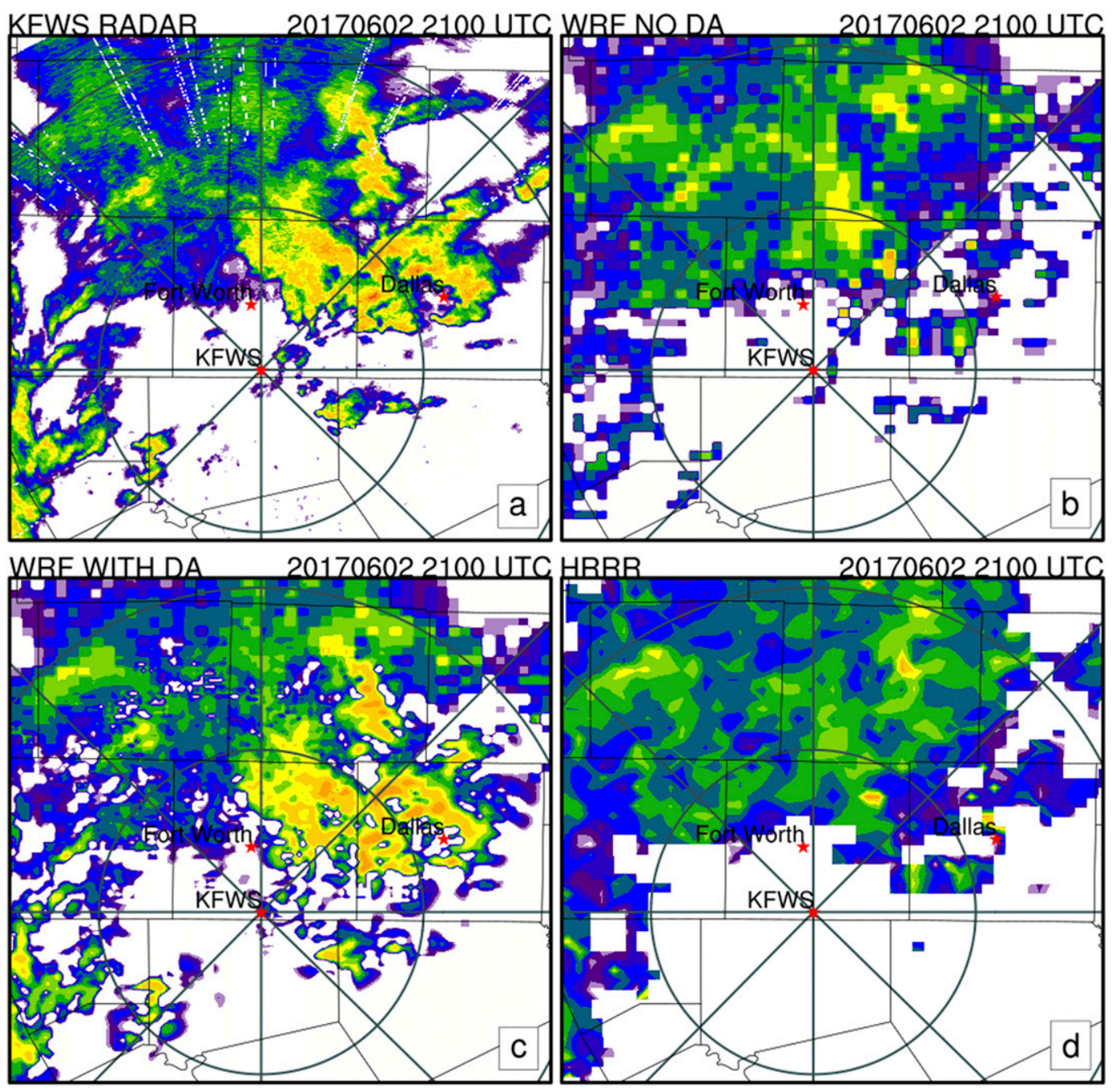

Reflectivity (dBZ) at $1 \mathrm{~km}$ height

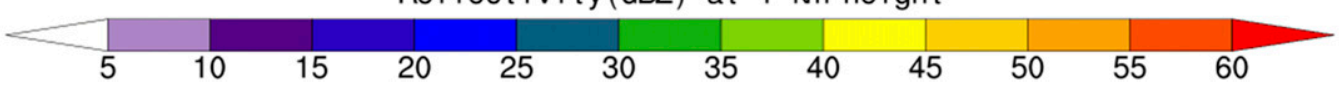

FIG. 5. Reflectivity map from (a) NEXRAD KFWS radar observed, (b) initial condition to WRF from HRRR model analysis, (c) WRF-3DVAR analysis with radar observations and first guess from HRRR model analysis in (b), and (d) 3-km-resolution HRRR model analysis.

precipitation events. However, these desirable values will be tested in the future when CASA implements the realtime blended nowcast.

\section{Results and discussion}

a. Evaluation of CASA's real-time operational reflectivity nowcast system

Figure 3 displays a comparison of the spatial reflectivity map of CASA and NEXRAD KFWS radarobserved merged reflectivity (top row) and DARTS nowcast (bottom row) reflectivity. The figure shows the single DARTS nowcast cycle started at 2100 UTC
2 June 2017 with the lead time of 10, 20, and $30 \mathrm{~min}$. Based on the data in this figure, DARTS can accurately nowcast the storm structure and intensity close to observation. Figure 4 shows DARTS nowcast skill scores averaged over all the nowcast cycles and precipitation types. DARTS has excellent prediction skills in supercell events, and the average CSI, POD, and ETS at the lead time of $30 \mathrm{~min}$ are $0.6,0.78$, and 0.45 , respectively. In line events, DARTS performed well until the 15-min lead time point, and the average CSI, POD and ETS are 0.4, 0.5, and 0.35 at the end of a 30-min lead time. In multicell events, DART had decent skill until $10 \mathrm{~min}$ and averaged CSI, POD, and 

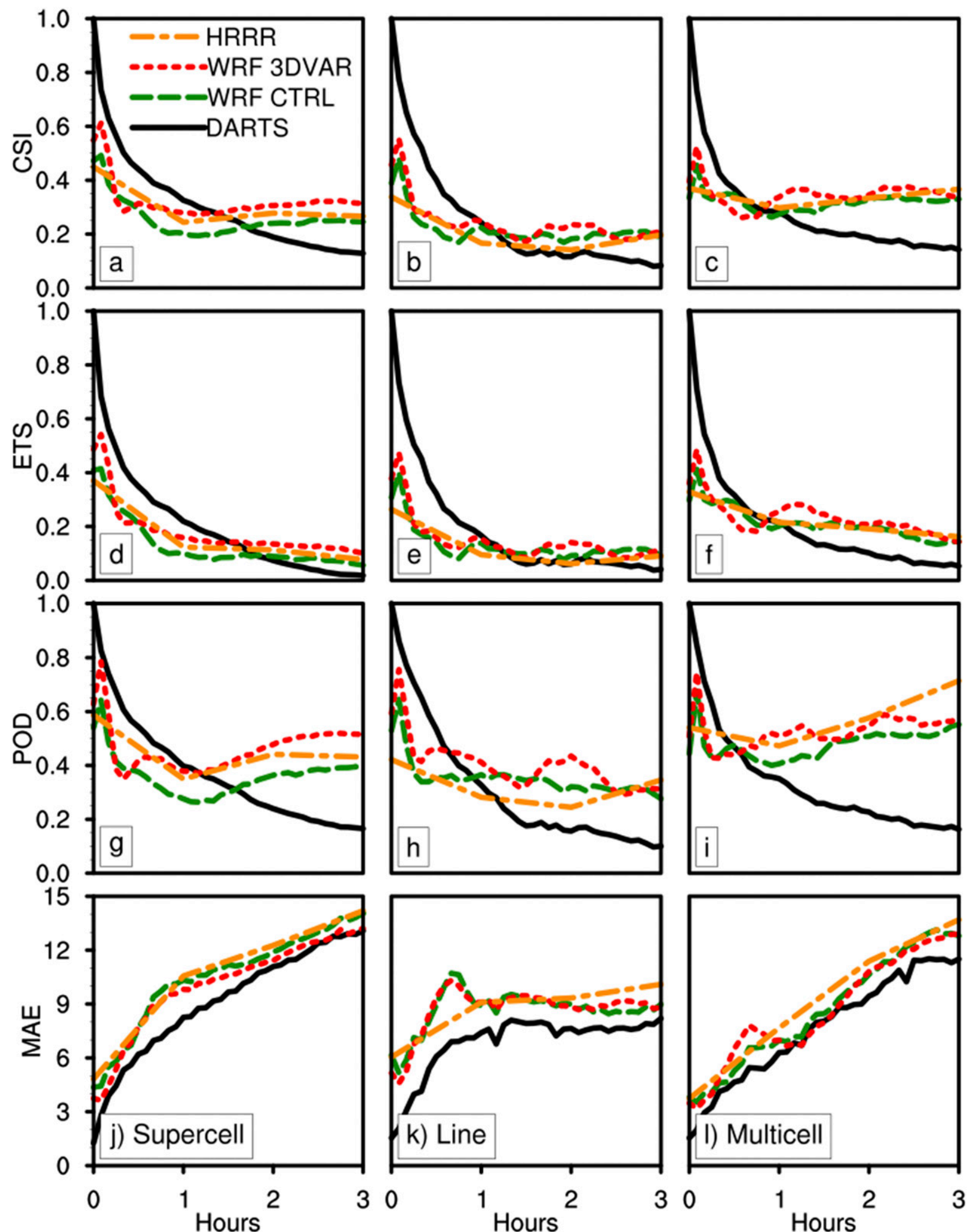

FIG. 6. Precipitation events and type averaged skill scores from DARTS nowcast, WRF CTRL, WRF-3DVAR prediction, and HRRR. 

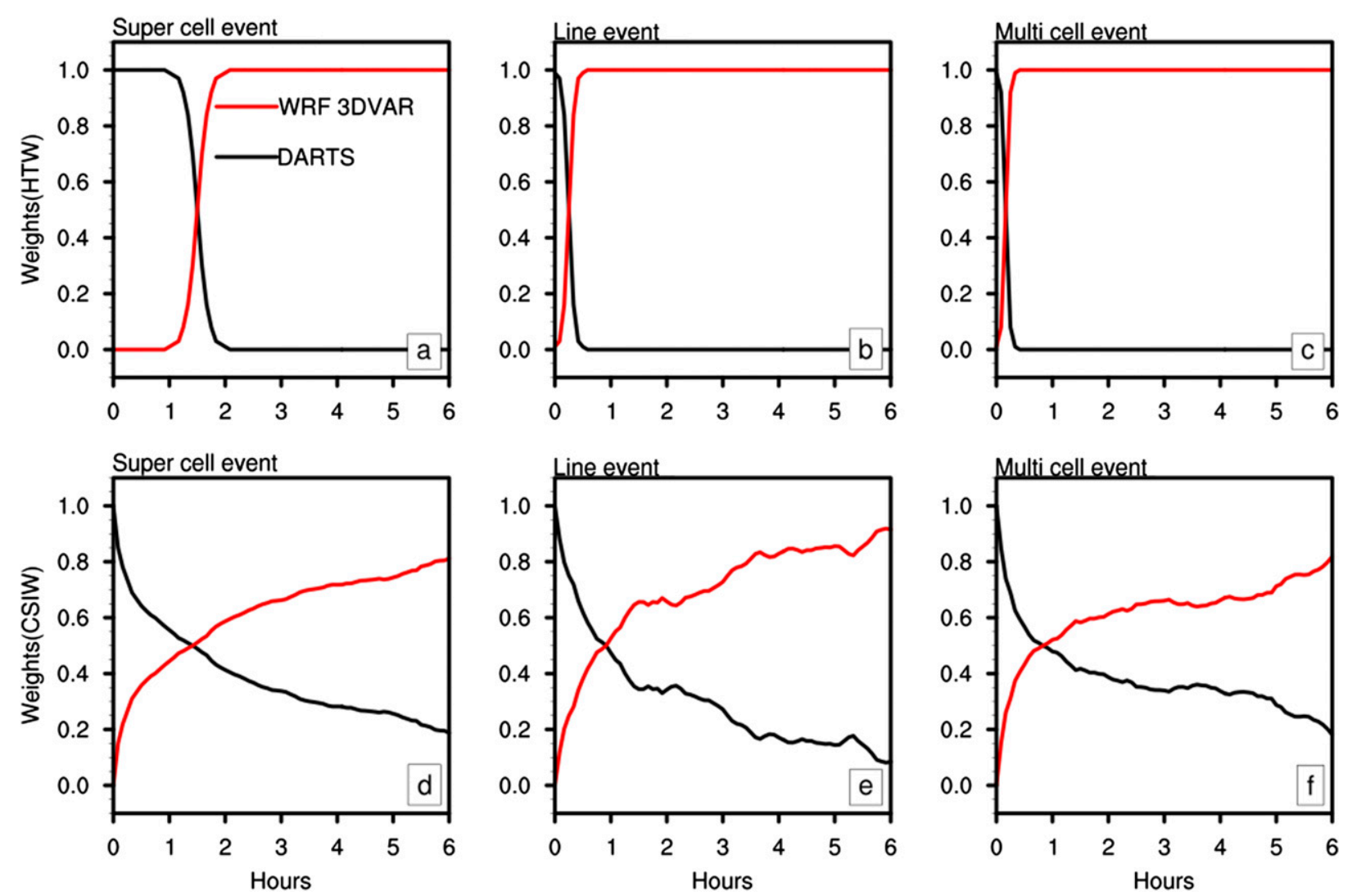

FIG. 7. Time-varying weights for DARTS nowcast and WRF-3DVAR prediction from (a)-(c) hyperbolic tangent curve weight (HTW) method and (d)-(f) critical success index weight (CSIW) method for three precipitation types.

ETS were $0.28,0.4$, and 0.2 at the end of 30-min lead time. The averaged MAE of all three types of precipitation events was less than $7 \mathrm{dBZ}$ until the lead time of $30 \mathrm{~min}$.

\section{b. Evaluation of DARTS, WRF, and HRRR model simulations}

Figure 5 shows the reflectivity map from (Fig. 5a) NEXRAD KFWS radar observation, (Fig. 5b) WRF initial conditions (first guess to 3DVAR assimilation) from HRRR model analysis, (Fig. 5c) WRF-3DVAR analysis after assimilation of radar observations, and (Fig. 5d) HRRR model analysis for a supercell event at 2100 UTC 2 July 2017. From the figure, it is seen that the assimilation of high-resolution radar data places the storm at the right location and adjusts the storm intensity, which was observed by ground radar. The WRF control, which initialized from the HRRR model, captured the small-scale features of the storm but underestimated the intensity. The HRRR model analysis captured the storm structure, but could not resolve the small-scale storm features and also underestimated the storm intensity. A similar sort of performance is seen from all the simulations conducted in this study. The overall results showed that the assimilation of high-resolution radar observations in the high-resolution WRF Model would improve the WRF Model's initial conditions regarding storm position and intensity.

Figure 6 shows the average skill score for DARTS, WRF control, WRF-3DVAR, and HRRR model simulations for all three precipitation types. The HRRR model simulations are available in 3-km resolutions, so all model forecasts were regrid into the HRRR model grid, and the skill scores were estimated. The model simulations were conducted to a 6-h lead time but the figure shows only 3-h for the purpose of clear visualization. The DARTS performed better than all WRF-based models until $1 \mathrm{~h} 30 \mathrm{~min}$, after that, the WRF-based models performed better than DARTS. The results show that the assimilation of highresolution radar observations enhances the accuracy of the model's initial conditions. The skill score of the best analysis from WRF-3DVAR is better than the WRF control run, which is used as the first guess in assimilation. The simulations initialized from the WRF- 

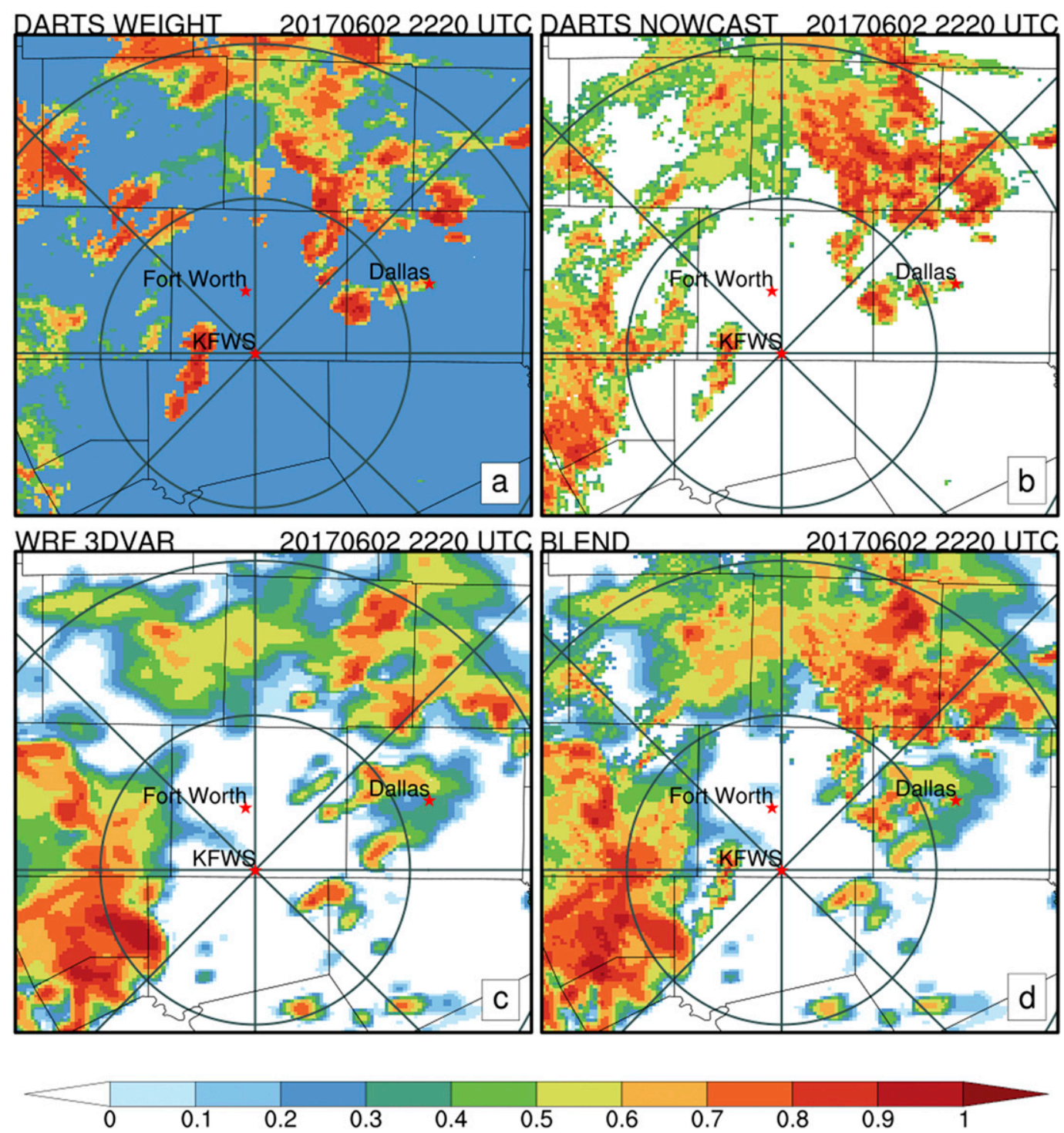

FIG. 8. (a) Two-dimensional weight estimated from salient cross dissolve (Sal CD) weight method, (b) normalized reflectivity from DARTS nowcast, (c) normalized reflectivity from WRF-3DVAR prediction, and (d) normalized reflectivity from DARTS nowcast and WRF-3DVAR prediction Sal CD blending method for 1-h lead time.

3DVAR best analysis have a better skill score than both the WRF control simulations and HRRR model. The simulation results from the WRF3DVAR and HRRR model shows the impact of assimilating the high-resolution radar observations into the high-resolution $(1 \mathrm{~km})$ WRF Model. In this study, even the 3DVAR assimilation system uses the first guess from previous WRF simulation (a warm start), but it is used in only three assimilation cycles. Nevertheless, the results are better than the HRRR model analysis, which used rapid-update cycles to assimilate all available observations in 15-min intervals. The WRF-3DVAR performance is supposed to be improved further by using rapid refresh assimilation cycles in real time.

\section{c. Weights generation for DARTS and WRF-3DAVR models}

Figures $7 \mathrm{a}-\mathrm{c}$ show the weights for DARTS and WRF3DVAR models from the HTW method. The weights from these methods are only a function of time, with the DARTS model weights higher for the first $1 \mathrm{~h}$ and $30 \mathrm{~min}$ of the forecast lead time. For supercell events, the HTW method gives higher weights to DART for the first $1 \mathrm{~h}$ and $30 \mathrm{~min}$. After that, the DARTS weight starts to decrease slowly and reaches zero at the lead time of $2 \mathrm{~h}$. 


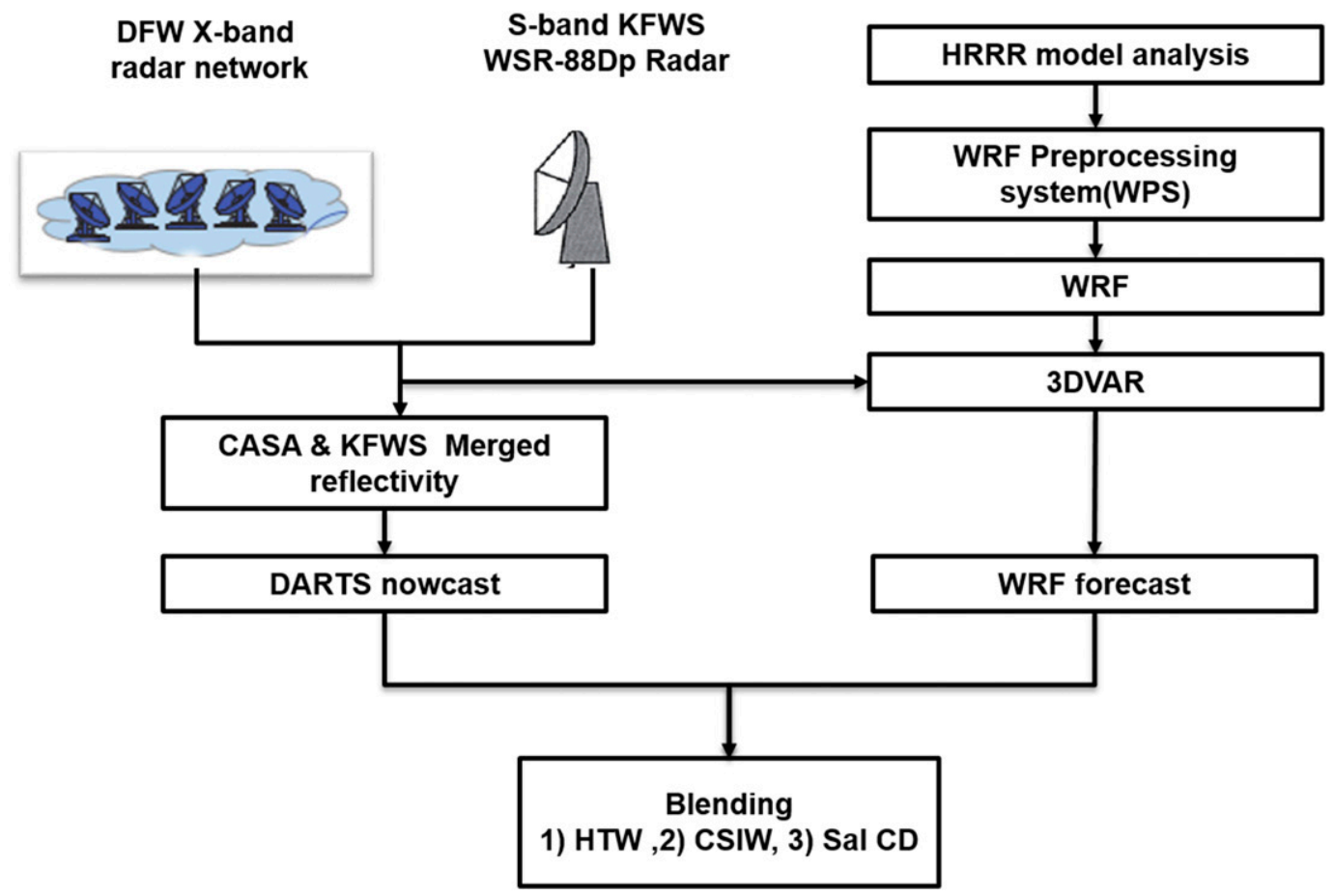

FIG. 9. Flowchart of DARTS nowcast and WRF-3DVAR model prediction blending method.

The blending only depends on the WRF-3DVAR model after the lead time of $2 \mathrm{~h}$. For line events, the HTW method gives higher weights to DARTS until $15 \mathrm{~min}$, and it rapidly reaches zero at $35 \mathrm{~min}$. In multicell events, DARTS has higher weight until $10 \mathrm{~min}$, and it becomes zero at 20-min lead time. The desirable parameters used in the HTW method for all three types of precipitation events is listed in Table 2. These parameters are determined through sensitivity studies. In the CSIW method, the parameter $\tau$ is estimated from the performance of DARTS and WRF on the nine precipitation events considered in this study. The $\tau$ was chosen in such a way that both the DARTS and WRF weights are equal (0.5), where the CSI score of DARTS and WRF intercept in the previous analyses. The estimated $\tau$ value for supercell events was 0.5293 , for line events was 0.5136 , and for multicell events was 0.5611 . Figure 8 a showed the twodimensional DARTS nowcast weights determined from the Sal CD method at 1-h forecast lead time (2220 UTC 2 June 2017), when DARTS and WRF-3DVAR have nearly equal weights. It clearly shows the weights vary spatially and values are a function of both DARTS and WRF-3DVAR predicted storm intensity. The normalized reflectivity map from DARTS nowcast (Fig. 8b) and WRF-3DVAR (Fig. 8c) also shows that higher weights are given to the pixels where DARTS has more extreme intensity than WRF-3DVAR. Figure 8d shows the normalized blended reflectivity from Sal CD weight method, and it also shows this method can retain the high-intensity pixels from both models.

\section{d. Evaluation of blending simulations}

Figure 9 shows the data flow of the blending procedure followed in this study. Figure 10a shows the reflectivity map from the CASA and NEXRAD KFWS radar merged observations. Figures $10 \mathrm{~b}$ and $10 \mathrm{c}$ show the reflectivity nowcast from DARTS and WRF3DVAR at the lead time of $1 \mathrm{~h} 30 \mathrm{~min}$. The data show that both models can capture the storm structure with a spatial and temporal shift from observations. Figures 10d-f show the blended reflectivity map from HTW, CSIW, and Sal CD blending methods for a supercell event at 2230 UTC 2 June 2017 . The $1 \mathrm{~h} 30 \mathrm{~min}$ lead time was chosen to compare the different weighting methods generating reflectivity when DARTS and WRF-3DVAR are in equal weights. It clearly shows both HTW and CSIW methods could not retain the high-intensity storm pixels, it only made a simple linear average of DARTS and WRF-3DVAR simulated reflectivity. The blended reflectivity from the Sal $\mathrm{CD}$ weighting method did retain the high-intensity storm pixels.

Figure 11 shows the averaged skill scores from the DARTS, WRF-3DVAR, and three blending methods for all precipitation types. The skill scores were estimated for every precipitation event (nine events) and 

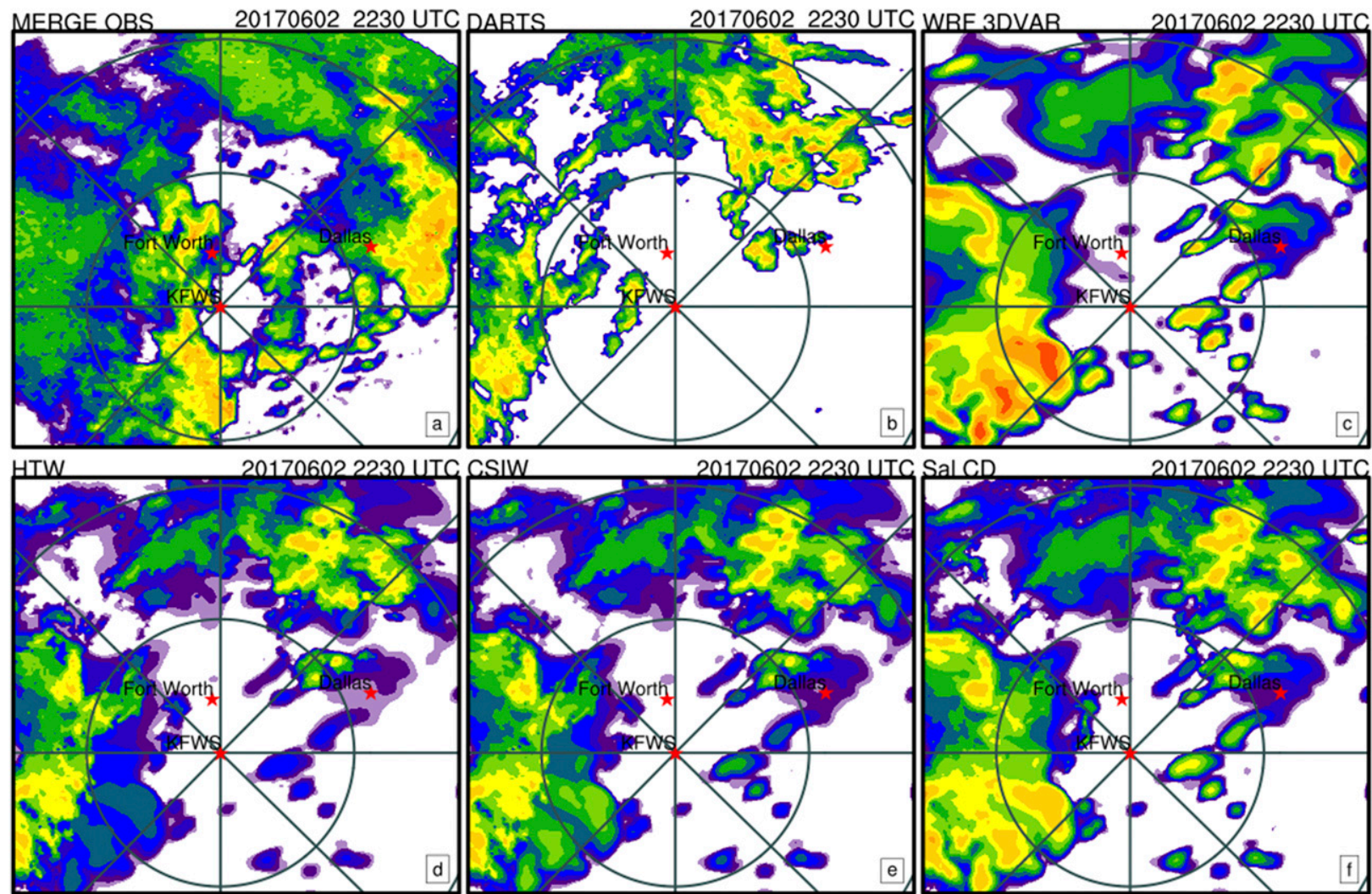

Reflectivity (dBZ)

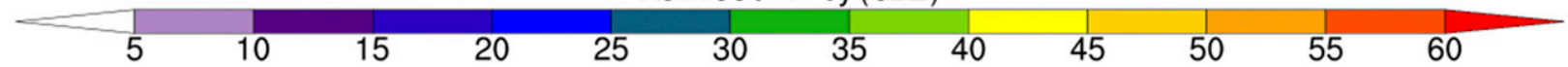

FIG. 10. Reflectivity map from (a) CASA and NEXRAD KFWS merged observation, (b) DARTS nowcast, (c) WRF-3DVAR prediction, blended simulation from (d) hyperbolic tangent curve weight (HTW) method, (e) critical success index weight (CSIW) method, and (f) salient cross dissolve (Sal CD) weight method for the lead time of $1 \mathrm{~h} 30 \mathrm{~min}$.

averaged within the precipitation type. For example, the time series of averaged skill scores for the supercell case shown in the figure were estimated from the average of five supercell events. In line and multicell cases, only two events are averaged. From the figure, it is clearly seen that the blended forecasts have better skills than the WRF-3DVAR forecast alone for the first few hours of lead time in all precipitation types. After a few hours, the skill score of blended forecasts are close to the WRF3DVAR model's skill, but better than the DARTS nowcast skill. This clearly shows that the blended forecast has the advantage over both DARTS nowcasts and WRF-3DVAR forecasts.

Table 3 lists the precipitation type and lead time average ( $30 \mathrm{~min}$, and 1,2 , and $6 \mathrm{~h}$ ) skill scores. Here, the skill score averages are found in two steps. In the first step, the skill scores are averaged within the precipitation types. In the second step, the cumulative averages are found between $5 \mathrm{~min}$ and lead time. The zero lead time is avoided because the DARTS extrapolation and three blending forecasts are the same as radar observations at zero lead time, this is not in the case for WRF3DVAR. At 30-min lead time, the DARTS and all blended simulations for all precipitation types performed better than WRF-3DVAR alone. The skill scores from the HTW method were exactly the same as DARTS in the supercell event, this was because the DARTS weighted fully until $1 \mathrm{~h} 30 \mathrm{~min}$ in HTW blending. The HTW method had worse skills in the line and multicell events than DARTS. In terms of line and multicell events, the CSIW method had higher skill scores than the other two methods in terms of ETS and MAE. The same level of performance was observed in the 1-h lead time average skill score. The HTW-method skill scores were identical with DARTS in supercell events, while the CSIW method had better performance in line and multicell events. From 2-h lead time onward, the blending simulations started performing better than DARTS for all precipitation events. The CSIW method had good performance in terms of MAE score and Sal 

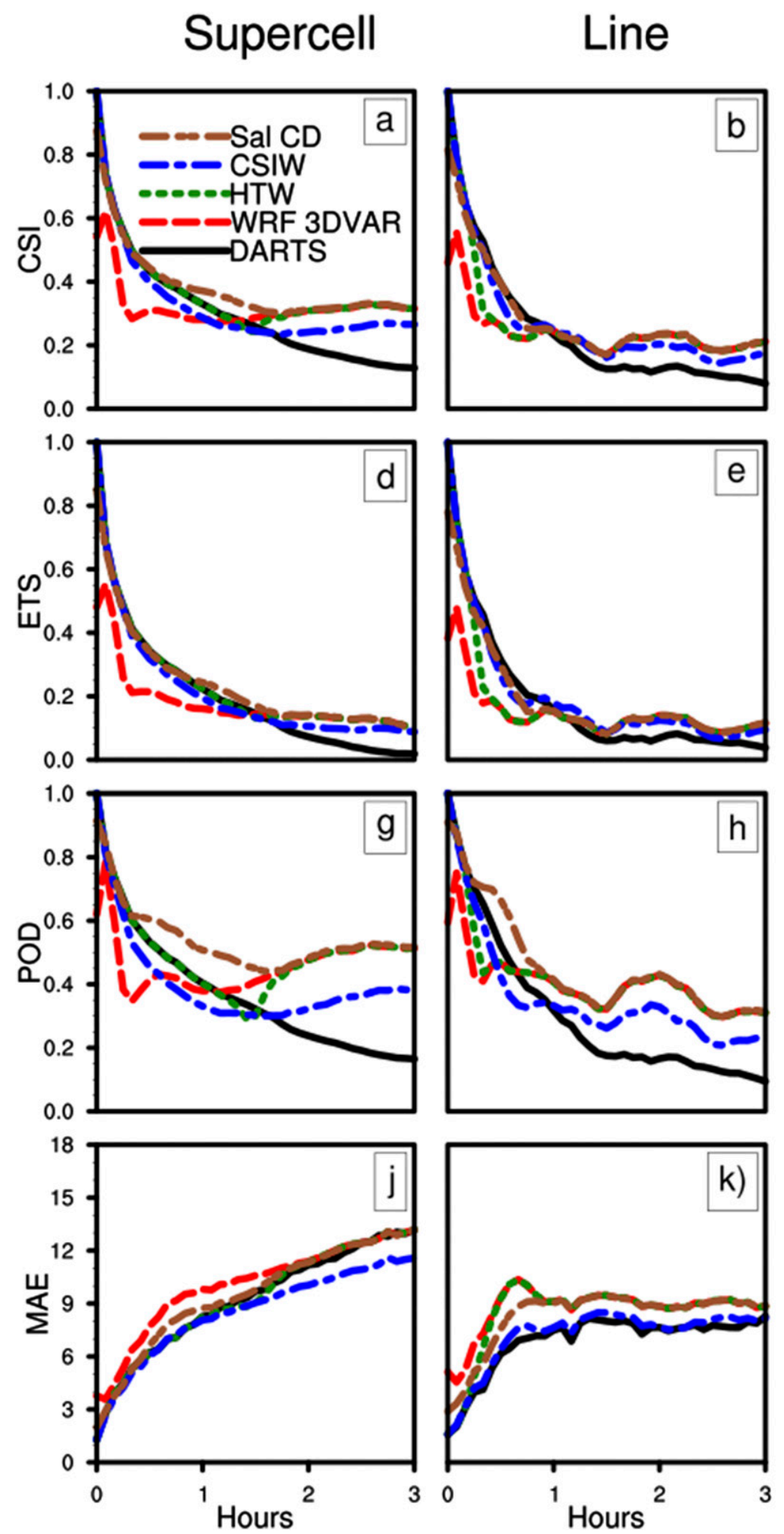

Multicell
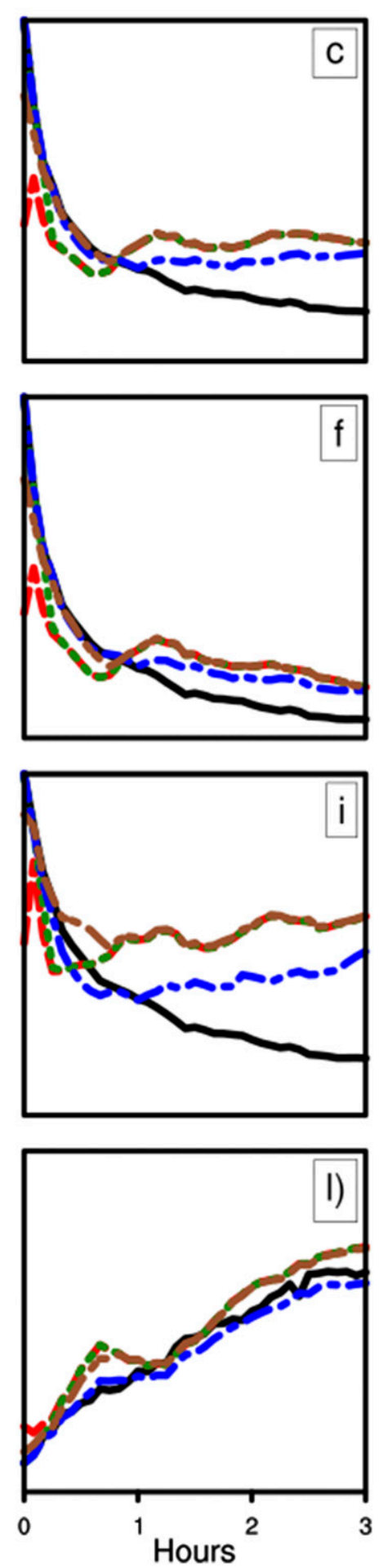

FIG. 11. Averaged skill scores (within precipitation type) from DARTS nowcast, WRF-3DVAR prediction, and three blending methods. 
TABLE 3. Averaged skill scores from within precipitation type and cumulative lead time.

\begin{tabular}{|c|c|c|c|c|c|c|c|c|c|c|c|c|c|}
\hline \multirow[b]{2}{*}{ Lead time } & \multirow[b]{2}{*}{ Model/event } & \multicolumn{4}{|c|}{ Supercell } & \multicolumn{4}{|c|}{ Line } & \multicolumn{4}{|c|}{ Multicell } \\
\hline & & CSI & POD & ETS & MAE & CSI & POD & ETS & MAE & CSI & POD & ETS & MAE \\
\hline \multirow[t]{5}{*}{$30 \mathrm{~min}$} & DARTS & 0.53 & 0.63 & 0.45 & 4.97 & 0.53 & 0.65 & 0.46 & 4.46 & 0.48 & 0.59 & 0.44 & 3.71 \\
\hline & WRF-3DVAR & 0.38 & 0.48 & 0.3 & 6.08 & 0.33 & 0.51 & 0.24 & 7.37 & 0.35 & 0.49 & 0.3 & 4.94 \\
\hline & HTW & 0.53 & 0.63 & 0.45 & 4.97 & 0.43 & 0.57 & 0.35 & 6.29 & 0.41 & 0.52 & 0.36 & 4.65 \\
\hline & CSIW & 0.51 & 0.58 & 0.44 & 4.79 & 0.5 & 0.59 & 0.44 & 4.73 & 0.47 & 0.54 & 0.43 & 3.73 \\
\hline & Sal CD & 0.53 & 0.67 & 0.45 & 5.16 & 0.51 & 0.72 & 0.43 & 5.8 & 0.46 & 0.63 & 0.41 & 4.4 \\
\hline \multirow[t]{5}{*}{$1 \mathrm{~h}$} & DARTS & 0.45 & 0.54 & 0.36 & 6.22 & 0.41 & 0.52 & 0.34 & 5.74 & 0.39 & 0.48 & 0.34 & 4.68 \\
\hline & WRF-3DVAR & 0.34 & 0.44 & 0.24 & 7.65 & 0.29 & 0.47 & 0.19 & 8.38 & 0.33 & 0.49 & 0.27 & 5.98 \\
\hline & HTW & 0.45 & 0.54 & 0.36 & 6.22 & 0.34 & 0.5 & 0.25 & 7.8 & 0.36 & 0.51 & 0.3 & 5.82 \\
\hline & CSIW & 0.42 & 0.48 & 0.34 & 6.1 & 0.39 & 0.47 & 0.32 & 6.07 & 0.39 & 0.45 & 0.34 & 4.74 \\
\hline & Sal CD & 0.46 & 0.61 & 0.36 & 6.66 & 0.39 & 0.6 & 0.3 & 7.3 & 0.4 & 0.58 & 0.33 & 5.6 \\
\hline \multirow[t]{5}{*}{$2 \mathrm{~h}$} & DARTS & 0.35 & 0.43 & 0.25 & 8.05 & 0.28 & 0.36 & 0.21 & 6.7 & 0.3 & 0.37 & 0.24 & 6.48 \\
\hline & WRF-3DVAR & 0.32 & 0.43 & 0.19 & 9.16 & 0.25 & 0.42 & 0.16 & 8.73 & 0.34 & 0.51 & 0.25 & 7.34 \\
\hline & HTW & 0.37 & 0.47 & 0.26 & 8.01 & 0.28 & 0.44 & 0.19 & 8.43 & 0.35 & 0.52 & 0.27 & 7.26 \\
\hline & CSIW & 0.34 & 0.4 & 0.24 & 7.64 & 0.3 & 0.39 & 0.22 & 7.01 & 0.34 & 0.42 & 0.27 & 6.23 \\
\hline & Sal CD & 0.39 & 0.54 & 0.27 & 8.39 & 0.31 & 0.49 & 0.21 & 8.17 & 0.37 & 0.55 & 0.29 & 7.15 \\
\hline \multirow[t]{5}{*}{$6 \mathrm{~h}$} & DARTS & 0.18 & 0.23 & 0.09 & 11.15 & 0.13 & 0.16 & 0.09 & 8.46 & 0.19 & 0.23 & 0.11 & 8.87 \\
\hline & WRF-3DVAR & 0.32 & 0.51 & 0.14 & 11.66 & 0.19 & 0.31 & 0.1 & 9.54 & 0.34 & 0.54 & 0.2 & 9.35 \\
\hline & HTW & 0.34 & 0.52 & 0.16 & 11.26 & 0.2 & 0.31 & 0.12 & 9.43 & 0.34 & 0.55 & 0.2 & 9.32 \\
\hline & CSIW & 0.29 & 0.42 & 0.14 & 10.15 & 0.19 & 0.26 & 0.11 & 8.62 & 0.32 & 0.46 & 0.19 & 8.27 \\
\hline & Sal CD & 0.34 & 0.55 & 0.16 & 11.42 & 0.21 & 0.33 & 0.12 & 9.36 & 0.35 & 0.56 & 0.21 & 9.27 \\
\hline
\end{tabular}

CD had high skill values in all types of precipitation in terms of CSI, POD, and ETS. At 6-h lead time, the skill scores were close between all three blending techniques. The Sal CD and HTW methods were identical in terms of CSI and ETS score in supercell events and better than the CSIW method. In line events also, both methods have identical ETS scores but Sal CD had a better CSI score. In multicell events, Sal CD had better skill than the other two methods.

\section{Summary and conclusions}

This research study attempted to extend the CASA's prediction system to 6-h lead time through blending DARTS nowcast with the WRF Model forecast. Nine precipitation events were simulated and analyzed over the DFW urban area and classified into three categories of supercell, line, and multicell events. A detailed evaluation of CASA's real-time operational reflectivity nowcast system was presented. The results showed the DARTS nowcast had excellent nowcast skills for supercell events up until $1 \mathrm{~h} 30 \mathrm{~min}$. In line and multicell events, the DARTS had a high skill score only until 15-min lead time. The high-resolution dual-pol radar observations were assimilated into the WRF Model to reduce the uncertainty in the initial conditions of the model. The results showed that the assimilation not only corrected the storm locations and intensity at initial conditions, but also enhanced the overall model predictions. The results showed that the blending improved the prediction skills compared with radar nowcast and WRF-3DVAR forecasts alone after 2-h lead time. In the first 30-min forecast lead time, HTW had better skills than the other two in supercell cases. In the line and multicell cases, the CSIW method had better performance for 30-min and 1-h forecast lead time. However, since the main focus of this study was to extend the CASA prediction system lead time to $6 \mathrm{~h}$, the conclusions are based on the 6-h lead time averaged skill scores. At 6 -h averaged lead time, the results were mixed. The merits and demerits of these three methods should be considered before selecting the best method for future studies. The theoretical formulation of both HTW and Sal CD methods are very similar, except that $\mathrm{Sal} C D$ has spatial variability in weights. In the Sal CD method, spatial weights are estimated on the day of the storm, unlike fixed weights in HTW and CSIW methods. Also, the HTW and CSWI methods also suffer from a "half intensity" problem when any of the models underestimate the intensity. However, in the Sal CD method, the halfintensity problem is eliminated by retaining the highintensity pixels. At the same time, keeping the high intensities of both sources of the forecast can be more problematic than the half intensity issue because will over predict the intensity. Also, the Sal CD method does not satisfy the "no bias condition," whereas HTW and CSIW methods do satisfy the "no bias condition." With the consideration of all skill scores, merits and demerits of all three blending methods, the HTW method is recommended to conduct further studies on CASA's blended forecast system. Also, the 
results were based on a relatively small sample set (only 9 events), and more representative figures might be derived from a larger sample in the future.

Acknowledgments. This work was mainly supported by the Hazards SEES program (Grand AGS-1331572) of the U.S. National Science Foundation. The authors additionally recognize the NCAR/CISL supercomputing facilities. The radar operations were supported by NCTCOG and the large CASA team of engineers and scientists is responsible for the operation of the DFW radar network.

\section{REFERENCES}

Atencia, A., and I. Zawadzki, 2015: A comparison of two techniques for generating nowcasting ensembles. Part II: Analogs selection and comparison of techniques. Mon. Wea. Rev., 143 2890-2908, https://doi.org/10.1175/MWR-D-14-00342.1.

Benjamin, S. G., and Coauthors, 2016: A North American hourly assimilation and model forecast cycle: The Rapid Refresh Mon. Wea. Rev., 144, 1669-1694, https://doi.org/10.1175/ MWR-D-15-0242.1.

Blaylock, B. K., J. D. Horel, and S. T. Liston, 2017: Cloud archiving and data mining of High-Resolution Rapid Refresh forecast model output. Comput. Geosci., 109, 43-50, https://doi.org/ 10.1016/j.cageo.2017.08.005.

Brown, D., R. Brownrigg, M. Haley, and W. Huang, 2012: The NCAR Command Language (NCL) (version 6.0. 0). UCAR/NCAR Computational and Information Systems Laboratory, https:// doi.org/10.5065/D6WD3XH5.

Chandrasekar, V., H. Chen, and B. Philips, 2018: Principles of highresolution radar network for hazard mitigation and disaster management in an urban environment. J. Meteor. Soc. Japan, 96А, 119-139, https://doi.org/10.2151/jmsj.2018-015.

Cornford, D., 2004: A Bayesian state space modelling approach to probabilistic quantitative precipitation forecasting. J. Hydrol., 288, 92-104, https://doi.org/10.1016/j.jhydrol.2003.11.040.

Dance, S., E. Ebert, and D. Scurrah, 2010: Thunderstorm strike probability nowcasting. J. Atmos. Oceanic Technol., 27, 79-93, https://doi.org/10.1175/2009JTECHA1279.1.

Dixon, M., and G. Wiener, 1993: TITAN: Thunderstorm identification, tracking, analysis, and nowcasting-A radar-based methodology. J. Atmos. Oceanic Technol., 10, 785-797, https:// doi.org/10.1175/1520-0426(1993)010<0785:TTITAA > 2.0.CO;2.

Foresti, L., M. Reyniers, A. Seed, and L. Delobbe, 2016: Development and verification of a real-time stochastic precipitation nowcasting system for urban hydrology in Belgium. Hydrol. Earth Syst. Sci., 20, 505-527, https:// doi.org/10.5194/hess-20-505-2016.

Gao, J., and D. J. Stensrud, 2012: Assimilation of reflectivity data in a convective-scale, cycled 3DVAR framework with hydrometeor classification. J. Atmos. Sci., 69, 1054-1065, https:// doi.org/10.1175/JAS-D-11-0162.1.

Germann, U., and I. Zawadzki, 2002: Scale-dependence of the predictability of precipitation from continental radar images. Part I: Description of the methodology. Mon. Wea. Rev., 130, 2859-2873, https://doi.org/10.1175/1520-0493(2002)130<2859: $\mathrm{SDOTPO}>2.0 . \mathrm{CO} ; 2$

— , and — 2004: Scale dependence of the predictability of precipitation from continental radar images. Part II: Probability forecasts. J. Appl. Meteor., 43, 74-89, https://doi.org/10.1175/ 1520-0450(2004)043<0074:SDOTPO > 2.0.CO;2.

Haiden, T., A. Kann, C. Wittmann, G. Pistotnik, B. Bica, and C. Gruber, 2011: The Integrated Nowcasting through Comprehensive Analysis (INCA) system and its validation over the eastern Alpine region. Wea. Forecasting, 26, 166183, https://doi.org/10.1175/2010WAF2222451.1.

Handwerker, J., 2002: Cell tracking with TRACE3D-A new algorithm. Atmos. Res., 61, 15-34, https://doi.org/10.1016/S01698095(01)00100-4.

Heye, A., K. Venkatesan, and J. Cain, 2017: Precipitation nowcasting: Leveraging deep recurrent convolutional neural networks. Proc. Cray User Group, Redmond, WA, Cray User Group.

Hwang, Y., A. J. Clark, V. Lakshmanan, and S. E. Koch, 2015: Improved nowcasts by blending extrapolation and model forecasts. Wea. Forecasting, 30, 1201-1217, https://doi.org/ 10.1175/WAF-D-15-0057.1.

Istok, M. J., and Coauthors, 2009: WSR-88D dual-polarization initial operational capabilities. 25th Conf. on International Interactive Information and Processing Systems in Meteorology, Oceanography, and Hydrology, Phoenix, AZ, Amer. Meteor. Soc., 15.5, http://ams.confex.com/ams/pdfpapers/148927.pdf.

Johnson, J., P. L. MacKeen, A. Witt, E. D. W. Mitchell, G. J. Stumpf, M. D. Eilts, and K. W. Thomas, 1998: The storm cell identification and tracking algorithm: An enhanced WSR-88D algorithm. Wea. Forecasting, 13, 263-276, https://doi.org/ 10.1175/1520-0434(1998)013<0263:TSCIAT $>2.0$. CO;2.

Kilambi, A., and I. Zawadzki, 2005: An evaluation of ensembles based upon MAPLE precipitation nowcasts and NWP precipitation forecasts. 32nd Conf. on Radar Meteorology, Albuquerque, NM, Amer. Meteor. Soc., P3R.4, http:// ams.confex.com/ams/pdfpapers/96982.pdf.

Kober, K., G. Craig, C. Keil, and A. Dörnbrack, 2012: Blending a probabilistic nowcasting method with a high-resolution numerical weather prediction ensemble for convective precipitation forecasts. Quart. J. Roy. Meteor. Soc., 138, 755-768, https://doi.org/10.1002/qj.939.

Megenhardt, D., C. Mueller, S. Trier, D. Ahijevych, and N. Rehak, 2004: NCWF-2 probabilistic nowcasts. 11th Conf. on Aviation, Range and Aerospace Meteorology, Hyannis, MA, Amer. Meteor. Soc., 5.2, https://ams.confex.com/ams/pdfpapers/ 81993.pdf.

Parrish, D. F., and J. C. Derber, 1992: The National Meteorological Center's spectral statistical-interpolation analysis system. Mon. Wea. Rev., 120, 1747-1763, https://doi.org/10.1175/ 1520-0493(1992)120<1747:TNMCSS > 2.0.CO;2.

Powers, J. G., and Coauthors, 2017: The Weather Research and Forecasting Model: Overview, system efforts, and future directions. Bull. Amer. Meteor. Soc., 98, 1717-1737, https://doi.org/ 10.1175/BAMS-D-15-00308.1.

Reyniers, M., 2008: Quantitative Precipitation Forecasts Based on Radar Observations: Principles, Algorithms and Operational Systems. Institut Royal Météorologique de Belgique, 60 pp.

Rinehart, R., 1981: A pattern recognition technique for use with conventional weather radar to determine internal storm motions. Atmos. Technol., 13, 119-134.

and E. Garvey, 1978: Three-dimensional storm motion detection by conventional weather radar. Nature, $\mathbf{2 7 3}, 287-289$, https://doi.org/10.1038/273287a0.

Ruzanski, E., V. Chandrasekar, and Y. Wang, 2011: The CASA nowcasting system. J. Atmos. Oceanic Technol., 28, 640-655, https://doi.org/10.1175/2011JTECHA1496.1. 
Schmeits, M. J., K. J. Kok, D. H. Vogelezang, and R. M. van Westrhenen, 2008: Probabilistic forecasts of (severe) thunderstorms for the purpose of issuing a weather alarm in the Netherlands. Wea. Forecasting, 23, 1253-1267, https:// doi.org/10.1175/2008WAF2007102.1.

Seed, A., 2003: A dynamic and spatial scaling approach to advection forecasting. J. Appl. Meteor., 42, 381-388, https://doi.org/ 10.1175/1520-0450(2003)042<0381:ADASSA > 2.0.CO;2.

Shi, X., Z. Gao, L. Lausen, H. Wang, D.-Y. Yeung, W.-k. Wong, and W.-c. Woo, 2017: Deep learning for precipitation nowcasting: A benchmark and a new model. Neural Information Processing Systems Conf., Long Beach, CA, Neural Information Processing Systems Foundation, 5617-5627.

Sokol, Z., J. Mejsnar, L. Pop, and V. Bližňák, 2017: Probabilistic precipitation nowcasting based on an extrapolation of radar reflectivity and an ensemble approach. Atmos. Res., 194, 245257, https://doi.org/10.1016/j.atmosres.2017.05.003.

Turner, B., I. Zawadzki, and U. Germann, 2004: Predictability of precipitation from continental radar images. Part III: Operational nowcasting implementation (MAPLE). J. Appl. Meteor., 43, 231-248, https://doi.org/10.1175/1520-0450(2004) $043<0231$ :POPFCR $>2.0$.CO;2.

Tuttle, J. D., and G. B. Foote, 1990: Determination of the boundary layer airflow from a single Doppler radar. J. Atmos. Oceanic Technol., 7, 218-232, https://doi.org/10.1175/1520-0426(1990) 007<0218:DOTBLA $>2.0$.CO;2.

Wang, G., W.-K. Wong, Y. Hong, L. Liu, J. Dong, and M. Xue, 2015: Improvement of forecast skill for severe weather by merging radar-based extrapolation and storm-scale NWP corrected forecast. Atmos. Res., 154, 14-24, https://doi.org/ 10.1016/j.atmosres.2014.10.021.

Wang, Y., and Coauthors, 2017: Guidelines for nowcasting techniques. WMO Rep. 1198, 82 pp., https://library.wmo.int/opac/ doc_num.php?explnum_id $=3795$.

Wolfson, M. M., B. Forman, R. Hallowell, and M. Moore, 1999: The growth and decay storm tracker. Preprints, Eighth Conf. on Aviation, Range, and Aerospace Meteorology, Dallas, TX, Amer. Meteor. Soc., 58-62.

Wong, M., W. Wong, and E. S. Lai, 2006: From SWIRLS to RAPIDS: Nowcast applications development in Hong Kong. PWS Workshop on Warnings of Real-Time Hazards by Using Nowcasting Technology, Sydney, Australia, WMO.

Wong, W., L. H. Yeung, Y. Wang, and M. Chen, 2009: Towards the blending of NWP with nowcast-Operation experience in B08FDP. Symp. on Nowcasting, Whistler, BC, Canada, WMO, 4.5.

Woo, W., and W. Wong, 2017: Operational application of optical flow techniques to radar-based rainfall nowcasting. Atmosphere, 8, 48, https://doi.org/10.3390/atmos8030048.

Xingian, S., Z. Chen, H. Wang, D.-Y. Yeung, W.-K. Wong, and W.-c. Woo, 2015: Convolutional LSTM network: A machine learning approach for precipitation nowcasting. Neural Information Processing Systems Conf., Montreal, QC, Canada, Neural Information Processing Systems Foundation, 802-810.

Xu, K., C. K. Wikle, and N. I. Fox, 2005: A kernel-based spatiotemporal dynamical model for nowcasting weather radar reflectivities. J. Amer. Stat. Assoc., 100, 1133-1144, https:// doi.org/10.1198/016214505000000682. 